\title{
BIOLOGIA REPRODUTIVA DE JUMENTOS. I. BIOMETRIA TESTICULAR E COMPORTAMENTO SEXUAL DURANTE A COLHEITA DE SËMEN*
}

\author{
REPRODUCTIVE BIOLOGY OF DONKEY. I. TESTICULAR MEASUREMENT AND SEXUAL \\ BEHAVIOUR AT SEMEN COLLECTION
}

\author{
Rosana Noguelra de MORAIS'; Raul Gastūo MUCCIOL.O'; Wilson Gonçalve VIANA’
}

\begin{abstract}
RESUMO
Foram avaliadas as medidas testiculares $\mathrm{c}$ o comportamento sexual durante a colheita de sêmen de seis (6) jumentos da raça Pega, com idades variando entre $3 \mathrm{c} 9$ anos. Os valores médios obtidos para o comprimento, largura e altura testiculares foram, respectivamente, $10,12 \times 7,38 \times 7,68 \mathrm{~cm}$ para o testículo csquerdo c $10,35 \times 6,73 \times 7,12 \mathrm{~cm}$ para o dircito. Nåo foi encontrada diferença significante $(a=0,05)$ entre os volumes do testículo esquerdo e dircito de um mesmo reprodutor, cujos valores médios foram $201,36 \mathrm{~cm}^{3} \mathrm{e} 182.34 \mathrm{~cm}^{3}$. respectivamentc. Num total de 68 colheitas, o tempo de reaçăo médio (medido desde que o animal cra colkcado $\mathrm{cm}$ presença da fêmea até a ejaculaçăo) foi de 10,98 minutos, variando de dois a 45 min. (cocficiente de variação $=59 \%$ ). Durante o periodo de excitaçăo observaram-se reaçđes, consideradas por vários autores como características da espócie, tais

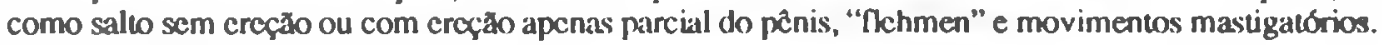

UNITERMOS: Reproduçăo, jumenıos; Biometria; Comportamento sexual

\section{INTRODUÇÃO}

Em funçăo de conhecida importância social c cconômica dos asininos ao longo do tempo e, principalmente, naqueles países e/ou regioes onde a agricultura ainda depende quase que cxclusivamente da traçăo animal, surge recentemente um grande interesse no aprimoramento ou adequação de técnicas reprodutivas já consagradas $\mathrm{cm}$ outras espécies para os jumentos. Frente a csta situação, propuscmo-nos estudar alguns paråmetros básicos para a seleçăo de reprodutores asininos a serem utilizados $\mathrm{cm}$ programas de inscminação artificial, incluindo a biometria testicular e o comportamento sexual durante a colheita de sêmen.

A avaliação dos testículos c bolsa testicular é um dos componentes fundamentais do exame andrológico, sendo que a biometria testicular vem sendo utilizada como ponto de partida para a cstimativa da fertilidade potencial de reprodutores bovinos.

De acordo com EL WISHY' (1974), existe uma correlação bastante significante entre as medidas de altura $(r=0,72)$ c volume $(r=0,70)$ testiculares com a reserva espermática gonadal de jumentos. Os valores de comprimento, largura c altura testiculares encontrados pelo mesmo foram ao redor de $8 \times 5 \times 5 \mathrm{~cm}$, respectivamente, sendo muito próximos daqueles encontrados por KREUCHAUF, 10 (1983, 1984) para as mesmas medidas $(8,5 \times 6 \times 6 \mathrm{~cm})$. Nåo foram detectadas diferenças significantes entre o volume dos testículos esquerdo $\mathrm{c}$ dircito de um mesmo reprodutor.

No Brasil foram encontrados valores ligeiramente superiores para o comprimento $(9,5 \mathrm{~cm})$ e largura $(6,0 \mathrm{~cm})$ de tesúculos de reprodutores asininos da raça Pega'.

Com relação à colheila de sêmen de jumento, a maioria dos autores concorda quanto ao uso da vagina artificial como método de cleiçăol, 2, 9, 13, 14, 17, 11, sendo muito variável o tipo de mancquim utilizado: jumentas e/ou éguas $\mathrm{cm}$ cio natural ${ }^{1,13}$ ou indurido 2,14, jumentas no diestro', jumentos ou pôneis castrados" e "phantom"11

Comparado a outras espécies domésticas, o jumento parece requerer um tempo de excitação pré-copulatório bem maior, podendo variar, em média de 10 a 30 minutos desde o contalo visual do reprodutor com o mancquim, até a ereçăo peniana completa, seguida de cópula c cjaculaçăo, 10, 11, 12, 13, 14, 16. Durante cste período parece ser comum a ocorréncia de saltos scm creçăo ou com creçào apenas parcial do péniss., 14, i6, sendo ainda descritas outras reaçös como "flehmen", obscrvável nos cquiinos, c movimentos mastigatórios como os descritos por VANDEPLASSCHE et al." (1981) para jumen-

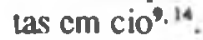

\section{MATERIAL E MÉTODO}

\section{Animais}

Foram utilizados seis (6) jumentos da raça Pega, pertencentes a três propricdades rurais do município de Săo Carlos - S.P., os quais foram designados por letras de $\mathrm{A}$ a $\mathrm{F}$. A altura e pesos médios dos animais eram de $1,30 \mathrm{~m} \mathrm{e} 400 \mathrm{~kg}$, respoctivamentc, $c$ a idade de cada um no início do expcrimento cra

1 - Professor Assistente - Universidade Fedenal do Parant

2 - Professor Associado - Frauldade de Medicina Veterinaria e Zootconia da USP

3 - Professor Doutor - Faculdade de Medicina Veterinaria e Zootecnia da USP

- Projeco fínenciado pela FAPESP - Fundaçio de Amparo a Pesquisa do listado de Sáo Paulo 
de 4 (A), 6 (B), 6 (C), 8 (D), 9 (E) e 3 (F) anos. Todos foram submetidos previamente a exame clínico e andrológico, tendo sido considerados aptos para o experimento. Durante todo o período experimental, de outubro de 1988 a abril de 1989, os animais foram mantidos $\mathrm{cm}$ piquetes individuais, com livre acesso às baias, recebendo raçăo c suplementaçăo mineral bàanceadas, com água à vontade.

\section{Biometria testicular}

Cada reprodutor foi submetido a três mensuraçōes testiculares, onde cada testículo foi medido individualmente, utilizando-se um paquímetro. Determinou-se o comprimento (crâniocaudal), a largura (médio-lateral e a altura (dorso-ventral) de cada testículo. Na determinaça do comprimento excluiu-se a cauda do epidídimo. Foram registradas as médias entre as tuês leituras.

Para fins comparativos entre os testículos dircito e esquerdo de um mesmo reprodutor, foi feita a estimativa do volume testicular, utilizando-se a equaçāo de regrcssão proposta por EL WISHY' (1974) a saber:

$Y_{v_{t}}=33,5757 . X-56,57$, onde $Y_{v t}=$ volume testicular e $X=$ altura do testículo $\mathrm{cm} \mathrm{cm}$.

Aproveitando os valores biométricos encontrados c com o intuito de comparaça entre os reprodutores, calculou-se o "índice testicular (IT)" proposto por KENNEY (1989)*, atravês da fórmula:

\section{$I T=\frac{(c \times 1 \times \text { a de } T E)+(C \times \perp \times \text { ado } T D)}{100}$}

onde $\mathrm{c}=$ comprimento, $1=$ largura $\mathrm{c}$ a $=$ altura de cada testículo $\mathrm{em} \mathrm{cm}$.

\section{Comportamento sexual}

Os animais foram submetidos a colheitas de sĉmen, numa frequência de dois ejaculados por semana, totalizando 68 amostras. A colheita foi realizada de acordo com a técnica preconizada para garanhoes ${ }^{15}$. Como mancquim foram utilizadas éguas em cio natural e, apenas para o jumento $\mathrm{C}$, empregou-se jumentas em estro, por se tratar de um reprodutor năo habituado a realizar cobertura de éguas.

O tempo despendido desde que o animal era colocado $\mathrm{cm}$ contato visual com a fêmea até a ejaculação, ao qual denominou-se tempo de reaçå, registrado $\mathrm{cm}$ minutos, foi observado, juntamente com outras reaçðes apresentadas pelos reprodutores.

\section{RESULTADOS}

O valor médio encontrado para as medidas de comprimento, largura e altura dos testículos, incluindo dircito e esquerdo foi $10,2 \times 7,0 \times 7,4 \mathrm{~cm}$. Os resultados individuais da biometria, assim como os índices testiculares calculados, sāo apresenta- dos na Tab. 1. Não foi constatada diferença significante (a = $0,05)$ entre os valores dos testículos esquerdo e dircito de um mesmo reprodutor.

Considerando-se o comportamento sexual, todas as tentativas de colheita de semen foram contempladas com pleno exito, embora o tempo de reaçăo tenha apresentado grande variaçåo. O valor mínimo para o tempo de reação foi de dois minulos para o jumento $\mathrm{E}$ c, o máximo, foi de 45 minutos, para o jumento $C$, com uma média global de $10,98 \pm 6,48$ minutos. Os valorcs médios individuais cstão aprescntados na 'Tab. 2. $O$ cocficiente de variaçăo para as 68 amostras foi de 59\%.

\section{DISCUSSĀO F CONCLUSŌES}

No presente experimento, as médias oblidas para as medidas Icsticulares foram superiores àquelas oblidas por EL WISHY' (1974) c KREUCHAUF9.10 ( 1983, 1984). Provavelmente outras variaçoes scjam devidas a diferenças entre o peso corporal múdio dos animais por nós estudados $(400 \mathrm{~kg})$ e o daqueles cstudados pelos referidos autores (257 kg E $125 \mathrm{~kg}$, respectivamente), uma vez que a faixa de idade năo variou muito. Tal fato fica ainda mais cvidente yuando constatamos diferenças mínimas entre os nossos achałos e ayueles obtidos com animais da inesma raça c peso semelhante ${ }^{3}$.

Concordando com os demais autores ${ }^{3.9}$. 10, nao foi detectada diferença significante entre o volume do testículo esquerdo e direito de um mesmo reprodutor.

Com relação ao índice testicular, os resultados por nós obtidos foram superiores an valor 8,0 , considerado ideal para garanhळes (KENNEY, 1989*). Houve uma cena uniformidade entre os reprodutores estudados, sendo que o menor valor correspondeu ao animal mais jovem, cujo desenvolvimento testicular provavelmente ainda năo tinha sido completado.

Apesar do número reduzido de animais avaliados, acreditamos que jumentos adultos da raça Pega, com desenvolvimento corporco normal, devam apresentar como medidas testiculares de comprimento, largura e altura, valores proximos a $10 \times 7 \times$ $7 \mathrm{~cm}$, respectivamente.

Consickrando-se a colheita de scemen, o uso da vagina artificial provou ser uma técnica de fácil accilaçà pelos jumentos. Com exceçăo do jumento E, nenhum dos animais havia sido submetido a colheita de sêmen antes do inf́cio do experimento. No entanto, apcnas um reprodutor năo accitou a vagina artificial na primcira tentativa e sim na terceira. Após essa fase de adaptaçăo, quando se fez necessário recorrer ao uso de tapaolhos para o referido animal, todos se mostraram bastante dóccis e de fácil mancjo, o que está de acordo com a maioria dos autores $1,2,9,13,14,17,19$.

Com relaçăo as éguas utilizadas como manequim, houve boa accitiçăo por parte dos jumentos, com exceçào do jumento $C$, o qual, durante sua fase de crescimento năo teve contato com éguas. Essas observaçoes corroboram o fato de que os jumenlos só accitam os diversos tipos de manequim $1.2,7,9,11,13,14$ se

- KENNEY, R. M. Falores que af alan a fertilidude do garanhIo. In: CONGRISSO I3RASIL.JiRO DE REPRODUÇAO ANIMAL, 8.,Belo Ilurizonte, 1989. (Comunicaçlo pessoal) 
MORAIS, R.N.; MUCCIOLO, R.G.; VIANA, W.G. Biologia reprodutiva de jumentos. I. Biometria testicular c compontamento sexual durante a colheita de sêmen. Braz. J. vet. Res. anlm. Scl., S.o Paulo, v. 30, n. 1. p. 47-50, 1993.

passarem por uma fase de adaptaçăo, principalmente quando jovens, segundo informaçøes dadas por criadores. ${ }^{12}$. Ou seja. para se conseguir bons resultados na colheila de sèmen de asininos, os animais devem ser cuidadosamente treinados c adaptados, tanto às condiçðes de técnica cm si, quanto ao tipo de manequim utilizado, o que implica na necessidade de um manejo correto, antes mesmo do início da vida reprodutiva.

Os resultados encontrados para o tempo de reaçăo aproximam-se daqueles relatados por alguns autores ${ }^{11,13.14}$, porém sฮ̃o muito menores do que os demais $2,1,9,16$. Apcsar das difercnças, de um modo geral, esses resultados demonstram que os jumentos, em comparacaá com outras espécies, realmente necessitam de um periodo de excitação pré-copulatória maior. É, provavelmente, uma característica cspecic-cspecífica, podendo sofrer influência de vários fatores tais como raça, idade e condicionamento reprodutivo de cada animal. Outro aspecto a ser considerado é que, apesar dos jumentos accitarem vários upos de manequim, o grau de atratividade que cada um cxcrce sobre os mesmos pode ser diferente e influir no tempo de reaçăo, o que explicaria, pelo menos $\mathrm{cm}$ partc, as diferenças entre nossos resultados e os dos demais autores.

Durante este período de excitaça e conte sexual notamos a ocortência de saltos sem ereçăo ou com ereçāo parcial do pênis, "flchmen" e movimentos mastigatórios, como os descritos para jumentas em cio". Essas observaçōes também foram fcitas por outros pesquisadores $\$$ 9.14, 16, o que nos lcva a acreditar que csses fenómenos façam parte do comportamento sexual normal dos jumentos, desde que naso influenciados pelo homem.

\section{SUMMARY}

Testicular measurements and sexual behaviour at semen collection of six donkeys of the "Pega" breed, ranging from 3 to 9 year of age were studied. The average testicular lenght, widht and height were, respectively, $10.12 \times 7.38 \times 7.68 \mathrm{~cm}$ for the left testicle and $10.35 \times 6.73 \times 7.12 \mathrm{~cm}$ for the right one. No statistical difference was found between left testicular volume $\left(x=201.36 \mathrm{~cm}^{3}\right)$ and right testicular volume $(x=$ $182.34 \mathrm{~cm}^{3}$ ) of the same sire. The reaction time (measured from the first visual contact with the female to cjaculation) ranged from 2 to 45 minutes and the overall mean was 10.98 $\pm 6.48 \mathrm{~min}(n=68)$. During the teasing period somc reactions, considered characteristic of donkeys by others investigators, was observed. These included mounting without penis erection or with partial penis erection, flehmen and mastigatory movements of the type described as an oestrous symptom in jennets.

UNITERMS: Reproduction, donkeys; Biomerry; Sex behavior

\section{REFERENCIAS BIBLIOGRÁFICAS}

01-BECK, E. EinfUhkung der Kunstlichen besamung in der maulierzucht Marokkos. Tierarztl. Umsch., v. 37, p. 21-4, 1982.

02-BIELANSKI, W.; WIERZBOWSKI, S. Some properties of the output of semen of jacks. Acta Blol. Cracov. S. Zool, v.5, p. 117-24, 1962.

03-COSTA, A.J.S.; VALE FILHO, V.R.; CASTRO, V.M. Biometria testicular e ańlise do sêmen do jumento Pega. Rev. bras. Reprod. anlm., p. 217, 1989. (Suplemento 1)

04-DOMINGUES, O. O jumento de Lagon Dourada. Publ. Inst. T.ootec., Rio de Janeiro, n.12, p. 1-64, 1963.

05-EL WISHY, A.B. Testicular and epididymal sperm reserves in the ass (Equus asinus) and stallion (Equus caballus). $\mathbf{Z}$. Tlerzuchtg. Zuchstbiol., v.91, p. 334-44, 1974.

06-IIAHN, J.; FOOTE, R.H.; SEIDEL, G.E. Testicular growth and rclated sperm output in dairy bulls. J. anlm. Scl., v. 29, p.41. 7. 1969.

07-HENRY, M.; GASTAL, E.L.; MEIRA, C.; DIAZ, A.P. Caracteristicas do sêmen de jumentos da raça Nordestina. In: CONGRESSO BRASILEIRO DE REPRODUCCÃO ANIMAL 7. Belo Horizonte, 1987. Programa. Colégio Brasileiro de Reproduçăo Animal, 1987. p. 72.

08-HENRY, M.; OLIVEIRA, M.M.F.; DIAZ, A.P.; GASTAL, E.L.; TOLENTINO, F.T. Comportamento de jumentos no período de cortejo e ato sexual. In: CONGRESSO BRASILEIRO DE REPRODUCÃO ANIMAL, 7., Belo Horizonte, 1987. Programa. Colégio Brasileiro de Reproduçzo Animal, Belo Horizonte, 1987. p. 71.

09-KREUCHAUF, A. Zum Fortpflanzungsgeschuhen bein Esel (Fquus asinus), Munchen, 1983. (Inaugural Dissertation zur Erlangung der Tiermedizinischen Doktorwurde Ticrarztlichen Facultat der Ludwing - Maximilians Universitat Munchen)

10-KREUCHAUF, A. Reproductive phisiology in the jackass. Anlm. Res. Develop., v. 20, p. 51-78, 1984.

11-KUHLMANN, J. Samenblologische, blochemische und cryotechnlsche Untersuchungen an eselsperma. Hannover, 1984. (Inaugural Dissertation zur Erlangung der tiermedizinischen Doktorwurde - Tierarztlichen Hoschule Hannover)

12-LEANDRO, P. R. Jumento Pega: o pai dos burros. Globo Rural, v. 2, n. 23, p. 26-33, 1987.

13-NISIIIKAWA, Y.; WAIDE, Y. Studies on reproduction in asses. VI - On the method of collection and the properties of semen. Bull. Nat. Inst. Agrke. Scl. G. . v. 1, p. 37-45, 1951 apud Anlm. Breed. Abst., v. 20, p.14, 1952. (resumo)

14-OSTROWSKI, J.E.B.; CORTESANO, J.A. Producción de mulares por inseminación artificial. I. Manejo del asno doador de semen. Rev. Med. Vet., Buenos Aires, v. 68, p. 210-6, 1987.

15-PICKETT, B.W.; SQUIRES, E. L.; McKINNON, A.O. Procedures for collection, evaluation and utillzation of stallion semen for artificial InsemInation. Fort Collins, Anim. Reprod. Lab., Colorado State University. 1987.

16.SCHAFER, $M$. Beobachtungen zum Paarungsverhalten des Rausescls (Equus asinus). Saugetlerck MItt, v. 30, p. 13-25, 1982 apud KREUCHAUF, $1984^{10}$, p. 52.

17. SHULLER BARBOSA, H.O. Observaçð̄es e sugestס̄es sobre a inseminação artificial em equídoos. Publ. Inst. Tootec, Rio de Janeiro, v. 10, p. 1-23, 1950.

18- SWIRE, P.W. Artificial insemination in mule production. In: MAULE, J.P., ed. The semen of animals and artinclal insemination. Bucks, Commonwealth Agricultural Bureaux, 1962. p. 298-303.

19 VANDEPLASSCHE, M.; WESSON, J.A.; GINTHER, O. J. Behavioral, follicular and gonadotropin changes during es. trous cycle in donkeys. Therlogenology, v. 16, p. 239-49, 1981.

Recebido para publicaçlo en 10/08/92 Aprovado para publiceflio em 15/12/92 
TABELA 1

Média (X) e desvio padrbo (DP) do comprimento (c), largura (l) e altura (a), em cm, e volume (v), em cms, de cada lestlculo, em jumentos, inctuindo os Indices testiculares (IT). S6o Cartos - SP, $1988 / 89$

\begin{tabular}{|c|c|c|c|c|c|c|c|c|c|}
\hline \multirow[b]{2}{*}{ Jumento } & \multicolumn{4}{|c|}{ Testlculo esquerdo } & \multicolumn{4}{|c|}{ Testlculo direito } & \multirow{2}{*}{$\begin{array}{l}\text { Indioe } \\
\text { Testiculer }\end{array}$} \\
\hline & c & I & $\mathbf{a}$ & $v$ & c & 1 & a & $v$ & \\
\hline $\begin{array}{l}A \\
B \\
C \\
D \\
E \\
F\end{array}$ & $\begin{array}{r}10,6 \\
10,3 \\
10,8 \\
10,1 \\
10,1 \\
8,8\end{array}$ & $\begin{array}{l}7,2 \\
8,4 \\
6,6 \\
6,6 \\
7,7 \\
7,7\end{array}$ & $\begin{array}{l}7,9 \\
7,7 \\
7,6 \\
7,6 \\
8,1 \\
7,4\end{array}$ & $\begin{array}{l}208,63 \\
201,92 \\
198,56 \\
191,85 \\
215,35 \\
191,85\end{array}$ & $\begin{array}{r}11,0 \\
10,3 \\
10,1 \\
10,2 \\
11,0 \\
9,5\end{array}$ & $\begin{array}{l}6,6 \\
6,5 \\
6,9 \\
6,5 \\
7,6 \\
6,3\end{array}$ & $\begin{array}{l}8,0 \\
7.8 \\
6.7 \\
7.1 \\
7.7 \\
5.4\end{array}$ & $\begin{array}{l}211,99 \\
205,28 \\
168,35 \\
181,78 \\
201,92 \\
124,71\end{array}$ & $\begin{array}{r}11,83 \\
11,88 \\
10,08 \\
9,71 \\
12,73 \\
8,24\end{array}$ \\
\hline $\begin{array}{r}\bar{X} \\
D P\end{array}$ & $\begin{array}{l}10,12 \\
\pm 0,70\end{array}$ & $\begin{array}{r}7,38 \\
\pm 0,68\end{array}$ & $\begin{array}{r}7.68 \\
\pm 0,28\end{array}$ & $\begin{array}{r}201,36 \\
\pm 36,38\end{array}$ & $\begin{array}{l}10,35 \\
\pm 0,58\end{array}$ & $\begin{array}{r}6,73 \\
\pm 0,47\end{array}$ & $\begin{array}{r}7,12 \\
\pm 0,97\end{array}$ & $\begin{array}{r}182,34 \\
\pm 32,58\end{array}$ & $\begin{array}{r}10,74 \\
\pm 1,68\end{array}$ \\
\hline
\end{tabular}

TABELA 2

Média $(\bar{X})$ e dosvio padrāo (DP) do tempo de reaçāo (min) de jumentos, com valoros máximos e minimos Sảo Carlos - SP, 1988/ 89.

\begin{tabular}{cccccc} 
Jumento & $\mathbf{n}$ & $\bar{X}$ & DP & Máximo & Mínimo \\
\hline A & 14 & $12,86^{c}$ & 5,01 & 20 & 05 \\
B & 14 & $10,14^{b i c}$ & 4,65 & 20 & 05 \\
C & 15 & $14.20^{c}$ & 9.79 & 45 & 06 \\
D & 06 & $14,33^{c}$ & 3,50 & 20 & 10 \\
E & 10 & $6,00^{\circ}$ & 3,23 & 14 & 02 \\
F & 09 & $7,33^{\circ .0}$ & 2,78 & 12 & 03 \\
\hline Total & 68 & 10,98 & 6,48 & 45 & 02 \\
\hline
\end{tabular}

$\mathbf{n}=$ número de cjaculados

$a, c=$ Médias com diferentes letras diferem significiuntementc $(\alpha=0,05)$ 